\title{
Status and Distribution of Potassium in Soils under Arecanut Gardens of Bhadra Command Area of Davanagere District, India
}

\author{
P. Amruthesh ${ }^{1}$, Parashuram Chandravamshi ${ }^{2 *}$, K.T. Gurumurthy ${ }^{1}$, L.B. Ashok ${ }^{3}$, \\ D.A. Sumana ${ }^{2}$ and Narayan S. Mavarkar ${ }^{4}$
}

${ }^{1}$ Department of Soil Science and Agricultural Chemistry, University of Agricultural and

Horticultural Sciences, Shivamogga, Karnataka, India

${ }^{2} Z A H R S$, Babbur farm, Hiriyur, UAHS, Shivamogga, Karnataka, India

${ }^{3}$ Department of Soil Science and Agricultural Chemistry, College of Horticulture, Hiriyur,

UAHS, Shivamogga, Karnataka, India

${ }^{4}$ Department of Agronomy, University of Agricultural and Horticultural Sciences, Shivamogga, Karnataka, India

*Corresponding author

\begin{tabular}{|c|c|}
\hline \multicolumn{2}{|r|}{ A B S T R A C T } \\
\hline & \multirow{5}{*}{$\begin{array}{l}\text { An investigation was carried out at College of Agriculture, Shivamogga during the year } \\
2016-17 \text { in order to know the status of potassium in soils under arecanut gardens of } \\
\text { Davanagere district, Karnataka. For the study, } 200 \text { surface and sub-surface samples were } \\
\text { collected from soils under arecanut garden of four taluks (Channagiri, Honnali, Harihara } \\
\text { and Davanagere) of Davanagere district. After processing, samples were analyzed for } \\
\text { potassium fractions. Results of the study indicated that, in surface and sub-surface soils of } \\
\text { different taluks, the mean water soluble potassium was highest in Channagiri taluk (18.93 } \\
\left.\mathrm{mg} \mathrm{kg}^{-1}\right) \text { and lowest in Davanagere taluk }\left(10.22 \mathrm{mg} \mathrm{kg}^{-1}\right) \text {, while the mean exchangeable } \\
\text { potassium was highest in Channagiri taluk }\left(127.89 \mathrm{mg} \mathrm{kg}^{-1}\right) \text { and lowest in Davanagere } \\
\left.\text { taluk (64.39 mg kg } \mathrm{kg}^{-1}\right) \text { and the mean non-exchangeable potassium was highest in } \\
\text { Channagiri taluk }(289.11 \mathrm{mg} \mathrm{kg} \text { ) and lowest in Davanagere taluk (137.93 mg kg-1). } \\
\text { Similarly, the mean lattice and total potassium was highest in Channagiri taluk }(3103.17 \\
\left.\left.\text { and } 3539.10 \mathrm{~m} \mathrm{~kg}^{-1}\right) \text { and lowest in Davanagere taluk (2077.11 and } 2289.65 \mathrm{mg} \mathrm{kg}^{-1}\right) \text {. }\end{array}$} \\
\hline & \\
\hline Article Info & \\
\hline $\begin{array}{l}\text { Accepted: } \\
\text { 04 November } 2 \\
\text { Available Onli } \\
10 \text { December } 2\end{array}$ & \\
\hline & \\
\hline
\end{tabular}

\section{Introduction}

The importance of potassium in the Indian agriculture needs no emphasis. It is a key nutrient which is required/removed from the soil in quantities comparable to or more than the nitrogen. The various forms of potassium in soil exist in equilibrium with one another and depletion of one form is replenished by other forms (Chandel et al., 1976). The removal of exchangeable $\mathrm{K}$ lead to release of the non-exchangeable fraction but in certain situation, the release rate may be insufficient to meet crop needs. In the nature, the potassium cycle consists of depletion from soil reserve on account of leaching losses beyond root zone, uptake and removal by the crops and addition through release of $\mathrm{K}$ from 
minerals, fertilizers and organic matter and debris. In plants, potassium helps in maintaining ionic balance in the cell, water relations and helps in root development. It is necessary for the formation of sugar, fat and fibrous materials and also favours early bearing. Knowing the fertilizer usage with regard to potassium, it can safely be said that now at least more than $50 \%$ of the soils will definitely require $\mathrm{K}$ application in order to give optimum crop yields (Tandon and Narayan, 1990). Therefore, an attempt has been made to study the different forms of potassium in soils under arecanut gardens of Bhadra command area of Davanagere district.

\section{Materials and Methods}

Characterization of surface soil $(0-15 \mathrm{~cm})$ and sub-surface soil $(15-30 \mathrm{~cm})$ was studied by taking fifty representative samples of arecanut gardens in each taluk of Channagiri, Honnali, Harihara, and Davanagere. Major land use pattern of these areas consists of intercropping of arecanut with banana, coconut and black pepper. The topsoil of these areas vary in colour which include brown, light and dark reddish brown, yellowish red, yellowish brown and black. The soils are gentle to moderately slope (3-10 \%) in nature with moderately well drained condition having acidic $\mathrm{pH}$ ranging from 4.5-6.5.

Soil samples were air-dried under shade and then powdered and sieved through $2 \mathrm{~mm}$ sieve and stored in clean polyethylene containers. Processed soil samples were analyzed in the laboratory for various physico-chemical parameters viz., the soil $\mathrm{pH}$ was measured in 1:2.5 soil water suspension using $\mathrm{pH}$ meter and EC $\left(\mathrm{dS} \mathrm{m}^{-1}\right)$ was measured in the supernatant solution of 1:2.5 soil water extract using conductivity bridge (Sparks, 1996). Organic carbon was estimated by Walkley and Black's wet oxidation method (Sparks, 1996). Exchangeable calcium and magnesium was determined by Versenate titration method (Black, 1965) and CEC by Neutral $N$ ammonium acetate saturation method (Page et al., 1982). Particle size distribution of soil samples were determined by International pipette method (Piper, 2002). Available potassium was extracted with neutral normal ammonium acetate extract and determined by using flame photometer as described by Jackson (1973). The laboratory analysis of soil samples was conducted at Agricultural and Horticultural Research station, Kathalagere, Davanagere, Karnataka during 2016-17.

\section{Results and Discussion}

Data on available and different fractions of potassium is presented in Table 1 to 4 .

\section{Channagiri}

Available potassium content ranged from 83.69 to 266.75 and 67.04 to $196.63 \mathrm{mg} \mathrm{kg}^{-1}$ in surface and sub-surface soils, respectively. Its content decreased with increase in soil depth. Water soluble potassium content ranged from 11.19 to 28.43 and 7.00 to $23.03 \mathrm{mg} \mathrm{kg}^{-1}$ in surface and sub-surface soils, respectively. The mean water soluble potassium content of Channagiri taluk was 18.93 and $13.95 \mathrm{mg} \mathrm{kg}^{-1}$ in surface and sub-surface soils, respectively. Exchangeable potassium content ranged from 72.50 to 254.75 and 59.00 to $189.63 \mathrm{mg} \mathrm{kg}^{-1}$ in surface and sub-surface soils, respectively. The mean exchangeable potassium content of Channagiri taluk was 127.89 and $90.88 \mathrm{mg}$ $\mathrm{kg}^{-1}$ in surface and sub-surface soils, respectively. The non-exchangeable potassium content varied from 258.00 to $310.00 \mathrm{mg} \mathrm{kg}^{-1}$ in surface soils whereas, in sub-surface soils it varied from 238.23 to $281.22 \mathrm{mg} \mathrm{kg}^{-1}$. Lattice form of K varied from 2649.18 to $3963.17 \mathrm{mg}$ $\mathrm{kg}^{-1}$ in the surface and 2353.90 to $3342.78 \mathrm{mg}$ $\mathrm{kg}^{-1}$ in the sub-surface soils. The total potassium content varied from 3054.01 to 4369.06 and 2753.41 to $3651.11 \mathrm{mg} \mathrm{kg}^{-1}$ in 
surface and sub-surface soils, respectively. The highest mean value $3539.10 \mathrm{mg} \mathrm{kg}^{-1}$ of total potassium was recorded in surface soils than in sub-surface soils $\left(3106.06 \mathrm{mg} \mathrm{kg}^{-1}\right)$ of Channagiri taluk.

\section{Honnali}

Available potassium content varied from 58.21 to 188.24 and 50.01 to $125.81 \mathrm{mg} \mathrm{kg}^{-1}$ in surface and sub-surface soils, respectively. The water soluble potassium ranged from 9.44 to 21.13 and 8.46 to $18.33 \mathrm{mg} \mathrm{kg}^{-1}$ in surface and sub-surface soils, respectively. The mean water soluble potassium content of Honnali taluk was $14.31 \mathrm{mg} \mathrm{kg}^{-1}$ and $12.13 \mathrm{mg} \mathrm{kg}^{-1}$ in surface and sub-surface soils, respectively. The data indicated that, the exchangeable potassium content ranged from 41.23 to 175.12 and 36.44 to $114.13 \mathrm{mg} \mathrm{kg}^{-1}$ in surface and sub-surface soils, respectively. The mean exchangeable potassium content of Honnali taluk was 113.99 and $81.10 \mathrm{mg} \mathrm{kg}^{-1}$ in surface and sub-surface soils, respectively. The highest mean value $173.40 \mathrm{mg} \mathrm{kg}^{-1}$ of nonexchangeable potassium was recorded in surface soils than in sub-surface soils (158.63 $\mathrm{mg} \mathrm{kg}^{-1}$ ) of Honnali taluk. The lattice potassium values of surface and sub-surface soils of Honnali taluk ranged from 2249.17 to $3587.88 \mathrm{mg} \mathrm{kg}^{-1}$ and 2131.00 to $3249.49 \mathrm{mg}$ $\mathrm{kg}^{-1}$ respectively. The highest mean value $2938.49 \mathrm{mg} \mathrm{kg}^{-1}$ of total potassium was recorded in surface soils than in sub-surface soils $\left(2876.79 \mathrm{mg} \mathrm{kg}^{-1}\right)$ of Honnali taluk.

\section{Harihara}

The data clearly noticed that the status of available potassium in Harihara taluk was found in the range 103.02 to $141.98 \mathrm{mg} \mathrm{kg}^{-1}$ and 76.70 to $109.09 \mathrm{mg} \mathrm{kg}^{-1}$ in surface and sub-surface soils, respectively. The water soluble potassium ranged from 8.23 to 22.20 and 6.11 to $17.32 \mathrm{mg} \mathrm{kg}^{-1}$ in surface and subsurface soils, respectively. The mean water soluble potassium content of Harihara taluk was 13.11 and $11.07 \mathrm{mg} \mathrm{kg}^{-1}$ in surface and sub-surface soils, respectively. Among all forms of potassium the water soluble potassium content found lowest in Harihara taluk.

The data indicated that, the exchangeable potassium content ranged from 93.31 to 132.33 and 66.00 to $95.46 \mathrm{mg} \mathrm{kg}^{-1}$ in surface and sub-surface soils, respectively. The mean exchangeable potassium content of Harihara taluk was 104.78 and $76.56 \mathrm{mg} \mathrm{kg}^{-1}$ in surface and sub-surface soils, respectively. The highest mean value $154.51 \mathrm{mg} \mathrm{kg}^{-1}$ of nonexchangeable potassium was recorded in surface soils than in sub-surface soils (142.30 $\mathrm{mg} \mathrm{kg}{ }^{-1}$ ) of Harihara taluk. The lattice potassium values of surface and sub-surface soils of Harihara taluk ranged from 2249.00 to 2987.00 and 2131.00 to $2792.96 \mathrm{mg} \mathrm{kg}^{-1}$ respectively. The highest mean value 2828.92 $\mathrm{mg} \mathrm{kg}{ }^{-1}$ of total potassium was recorded in surface soils than in sub-surface soils (2672.79 $\mathrm{mg} \mathrm{kg}{ }^{-1}$ ) of Harihara taluk.

\section{Davanagere}

The data clearly noticed that the status of available potassium in Davanagere taluk was found in the range 81.00 to 130.26 and 58.58 to $89.59 \mathrm{mg} \mathrm{kg}^{-1}$ in surface and sub-surface soils, respectively. The content of water soluble form of potassium in the surface layer of soil varied from 8.00 to $18.13 \mathrm{mg} \mathrm{kg}^{-1}$. Whereas, in sub-surface, the highest value of $14.13 \mathrm{mg} \mathrm{kg}^{-1}$ and lowest value of $6.45 \mathrm{mg} \mathrm{kg}$

${ }^{1}$ was noticed in soils of Davanagere. The content of exchangeable potassium in the surface layer of soil ranged from 73.00 to $112.13 \mathrm{mg} \mathrm{kg}{ }^{-1}$. In sub-surface soils, the highest value of $75.46 \mathrm{mg} \mathrm{kg}^{-1}$ and lowest value of $52.13 \mathrm{mg} \mathrm{kg}^{-1}$ was noticed in soils of Davanagere. The non-exchangeable potassium content varied from 95.94 to $285.90 \mathrm{mg} \mathrm{kg}^{-1}$ in surface soils. 


\begin{tabular}{|c|c|c|c|c|c|c|c|c|c|c|c|c|c|c|}
\hline \multirow{2}{*}{$\frac{v}{\text { ज्ञ }}$} & $\uparrow$ & 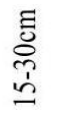 & 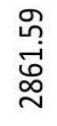 & 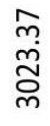 & $\begin{array}{l}\text { 으. } \\
\text { ญे } \\
\text { ర్లి }\end{array}$ & 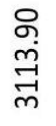 & 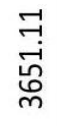 & $\begin{array}{l}\stackrel{d}{二} \\
\underset{m}{\vec{m}}\end{array}$ & 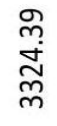 & 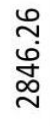 & $\begin{array}{l}\underset{\sigma}{\tilde{N}} \\
\stackrel{\text { N}}{\sim}\end{array}$ & 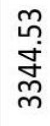 & 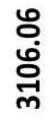 & 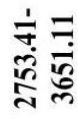 \\
\hline & & $\begin{array}{l}\frac{\text { D }}{0} \\
\frac{n}{0}\end{array}$ & 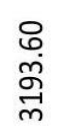 & $\begin{array}{l}\vec{N} \\
\stackrel{0}{0} \\
\stackrel{0}{0}\end{array}$ & 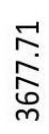 & $\begin{array}{l}\text { ọ } \\
\text { مे } \\
\text { लm }\end{array}$ & 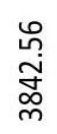 & 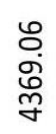 & 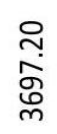 & 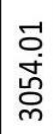 & 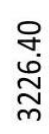 & 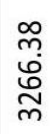 & 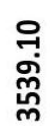 & $\begin{array}{l}\dot{1} \\
\dot{+} \\
\dot{0} \\
0\end{array}$ \\
\hline \multirow{2}{*}{ 光 } & & 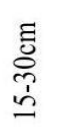 & $\begin{array}{l}\tilde{i} \\
\tilde{b} \\
\stackrel{0}{0}\end{array}$ & 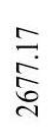 & के & $\begin{array}{l}\text { Oे. } \\
\text { ळे } \\
\text { ते }\end{array}$ & $\begin{array}{l}\stackrel{\infty}{\stackrel{i}{*}} \\
\underset{m}{m}\end{array}$ & $\begin{array}{l}\stackrel{m}{0} \\
\infty \\
\stackrel{\sim}{\sim}\end{array}$ & $\begin{array}{l}\tilde{b} \\
\ddot{d} \\
\approx \\
\hat{i}\end{array}$ & $\begin{array}{l}\vec{b} \\
\stackrel{2}{2} \\
\cdots\end{array}$ & $\begin{array}{l}8 \\
\text { ते } \\
\text { ते }\end{array}$ & $\begin{array}{l}\text { ते } \\
\text { ڤे }\end{array}$ & $\begin{array}{l}\stackrel{3}{0} \\
\stackrel{\infty}{*} \\
\stackrel{N}{*}\end{array}$ & 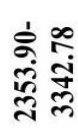 \\
\hline & & $\frac{\text { है }}{\stackrel{0}{\frac{1}{0}}}$ & 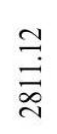 & $\begin{array}{l}\stackrel{\infty}{\infty} \\
\dot{\infty} \\
\underset{\sim}{\sim}\end{array}$ & $\begin{array}{l}\tilde{\Xi} \\
\stackrel{\infty}{m}\end{array}$ & $\begin{array}{l}\tilde{N} \\
\tilde{n} \\
\tilde{\delta} \\
\tilde{n}\end{array}$ & 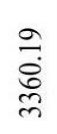 & $\begin{array}{l}\text { = } \\
\text { लू }\end{array}$ & 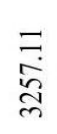 & $\begin{array}{l}\infty \\
\stackrel{a}{d} \\
\text { d. }\end{array}$ & 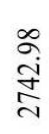 & $\underset{n}{\stackrel{n}{*}}$ & $\frac{\bar{c}}{\stackrel{\oplus}{m}}$ & $\begin{array}{l}\infty \\
0 \\
00 \\
0\end{array}$ \\
\hline \multirow{2}{*}{ 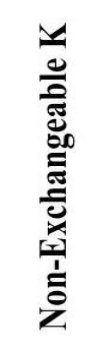 } & & $\begin{array}{l}\text { है } \\
\text { 仓े } \\
\stackrel{1}{\varrho}\end{array}$ & $\stackrel{\stackrel{\oplus}{ٍ}}{=}$ & $\frac{0}{\stackrel{a}{a}}$ & $\frac{\bar{\sigma}}{\vec{\sigma}}$ & 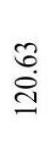 & $\stackrel{7}{2}$ & 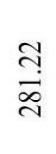 & $\begin{array}{l}\tilde{\imath} \\
\infty \\
\stackrel{\sim}{\sim}\end{array}$ & సે & $\begin{array}{l}\underset{f}{\infty} \\
\infty \\
\infty \\
\sim\end{array}$ & $\begin{array}{l}\hat{\sigma} \\
\infty \\
\tilde{j}\end{array}$ & $\begin{array}{l}\text { : } \\
\text { त̇ }\end{array}$ & $\begin{array}{l}\text { तु } \\
\text { స్ } \\
\text { స్ }\end{array}$ \\
\hline & $\stackrel{\infty}{\Xi}$ & 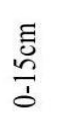 & 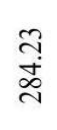 & $\begin{array}{l}\text { : } \\
\stackrel{8}{0}\end{array}$ & $\begin{array}{l}\widetilde{\infty} \\
\stackrel{\infty}{\infty} \\
\stackrel{\sim}{\infty}\end{array}$ & 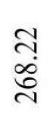 & 穴 & $\begin{array}{l}\text { oे } \\
\text { ¿ें }\end{array}$ & $\underset{m}{\stackrel{8}{\circ}}$ & 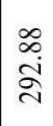 & 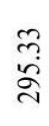 & $\begin{array}{l}\infty \\
0 \\
\infty \\
\infty \\
\sim\end{array}$ & $\begin{array}{l}= \\
\stackrel{0}{\circ}\end{array}$ & 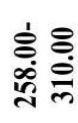 \\
\hline \multirow{2}{*}{ 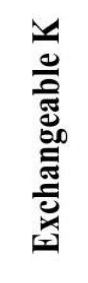 } & & 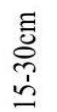 & $\frac{m}{3}$ & $\begin{array}{l}8 \\
\text { in }\end{array}$ & $\begin{array}{l}\text { J } \\
\text { ه }\end{array}$ & $\underset{\hat{3}}{\tilde{3}}$ & $\underset{\text { ळ. }}{=}$ & ڤn & ले & 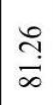 & $\frac{m}{2}$ & $\begin{array}{l}\Omega \\
\stackrel{0}{\infty}\end{array}$ & $\begin{array}{l}\infty \\
\stackrel{\leftrightarrow}{8} \\
\stackrel{8}{8}\end{array}$ & $\begin{array}{l}\text { के } \\
\text { in }\end{array}$ \\
\hline & & $\frac{\tilde{J}}{\frac{\tilde{D}}{3}}$ & $\stackrel{ \pm}{\circ}$ & 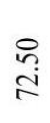 & $\begin{array}{c}\stackrel{0}{\infty} \\
\infty \\
\infty \\
\infty\end{array}$ & $\begin{array}{l}\stackrel{n}{n} \\
\end{array}$ & $\stackrel{n}{\stackrel{n}{\sigma}}$ & $\stackrel{\text { ণิ }}{\stackrel{\infty}{ }}$ & $\begin{array}{l}8 \\
\dot{0} \\
\stackrel{0}{0}\end{array}$ & ๙ૂ & $\begin{array}{l}\infty \\
\stackrel{\infty}{ \pm} \\
\stackrel{+}{I}\end{array}$ & 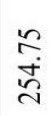 & $\begin{array}{l}\stackrel{a}{\leftrightarrows} \\
\stackrel{\Xi}{=}\end{array}$ & 空学 \\
\hline \multirow{2}{*}{ 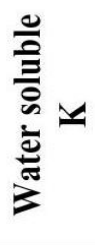 } & & $\begin{array}{l}\text { हू } \\
\text { 仓̦ } \\
\text { ஸ் }\end{array}$ & $\begin{array}{l}\Delta \\
\stackrel{d}{n}\end{array}$ & $\underset{\infty}{\mathbb{\infty}}$ & $\stackrel{m}{2}$ & $\vec{\circ}$ & 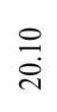 & 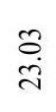 & $\stackrel{7}{a}$ & $\stackrel{8}{\stackrel{8}{-}}$ & $\stackrel{\stackrel{\infty}{=}}{=}$ & $\stackrel{8}{i}$ & $\stackrel{\leftrightarrow}{g}$ & 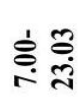 \\
\hline & & $\begin{array}{l}\frac{a}{0} \\
\frac{n}{c}\end{array}$ & $\begin{array}{l}\overline{\text { I }} \\
\text { ה. }\end{array}$ & $\stackrel{9}{\Xi}$ & $\begin{array}{l}\stackrel{\text { I }}{ \pm} \\
\end{array}$ & 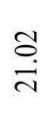 & $\underset{\substack{f \\
\infty}}{\stackrel{i}{d}}$ & $\begin{array}{l}8 \\
\text { ते }\end{array}$ & $\begin{array}{l}\text { oे } \\
\text { iे }\end{array}$ & $\begin{array}{l}8 \\
\infty \\
\infty\end{array}$ & 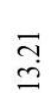 & 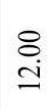 & $\underset{\infty}{\mathscr{\infty}}$ & 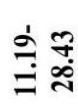 \\
\hline \multirow{2}{*}{ 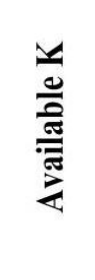 } & $\downarrow$ & $\begin{array}{l}\text { हूँ } \\
\text { 仓. } \\
\stackrel{n}{n}\end{array}$ & 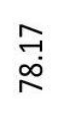 & 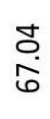 & $\begin{array}{l}\text { 공 } \\
\text { 号 }\end{array}$ & 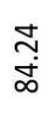 & $\begin{array}{l}\vec{\sim} \\
\stackrel{\rightrightarrows}{\exists}\end{array}$ & 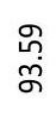 & 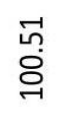 & $\begin{array}{l}2 \\
\infty \\
\infty\end{array}$ & $\underset{\tilde{I}}{\tilde{G}}$ & $\begin{array}{l}\tilde{O} \\
\dot{\phi} \\
\stackrel{\sigma}{\sigma}\end{array}$ & $\begin{array}{l}\infty \\
\stackrel{0}{0}\end{array}$ & 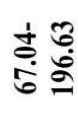 \\
\hline & & $\begin{array}{l}\text { है } \\
\frac{n}{0}\end{array}$ & $\stackrel{\stackrel{\sim}{\sim}}{\infty}$ & $\begin{array}{l}\stackrel{0}{0} \\
\ddot{\infty}\end{array}$ & $\begin{array}{l}\text { ํㅜㄹ } \\
\text { 유 }\end{array}$ & $\begin{array}{l}\text { กิ } \\
\text { హั }\end{array}$ & $\begin{array}{l}\infty \\
\stackrel{-}{-1} \\
\text { هి }\end{array}$ & શ્ণ & 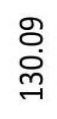 & 용 & $\begin{array}{l}8 \\
0 \\
\infty \\
\infty \\
\sim\end{array}$ & 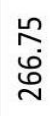 & $\begin{array}{l}\stackrel{0}{0} \\
\stackrel{\sigma}{\sigma}\end{array}$ & 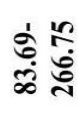 \\
\hline \multicolumn{2}{|c|}{$\dot{\infty} \dot{z}$} & & - & $\sim$ & $m$ & $\nabla$ & in & 0 & $r$ & $\infty$ & $a$ & 으 & $\sum_{\bar{\Sigma}}^{\bar{\Xi}}$ & 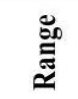 \\
\hline
\end{tabular}




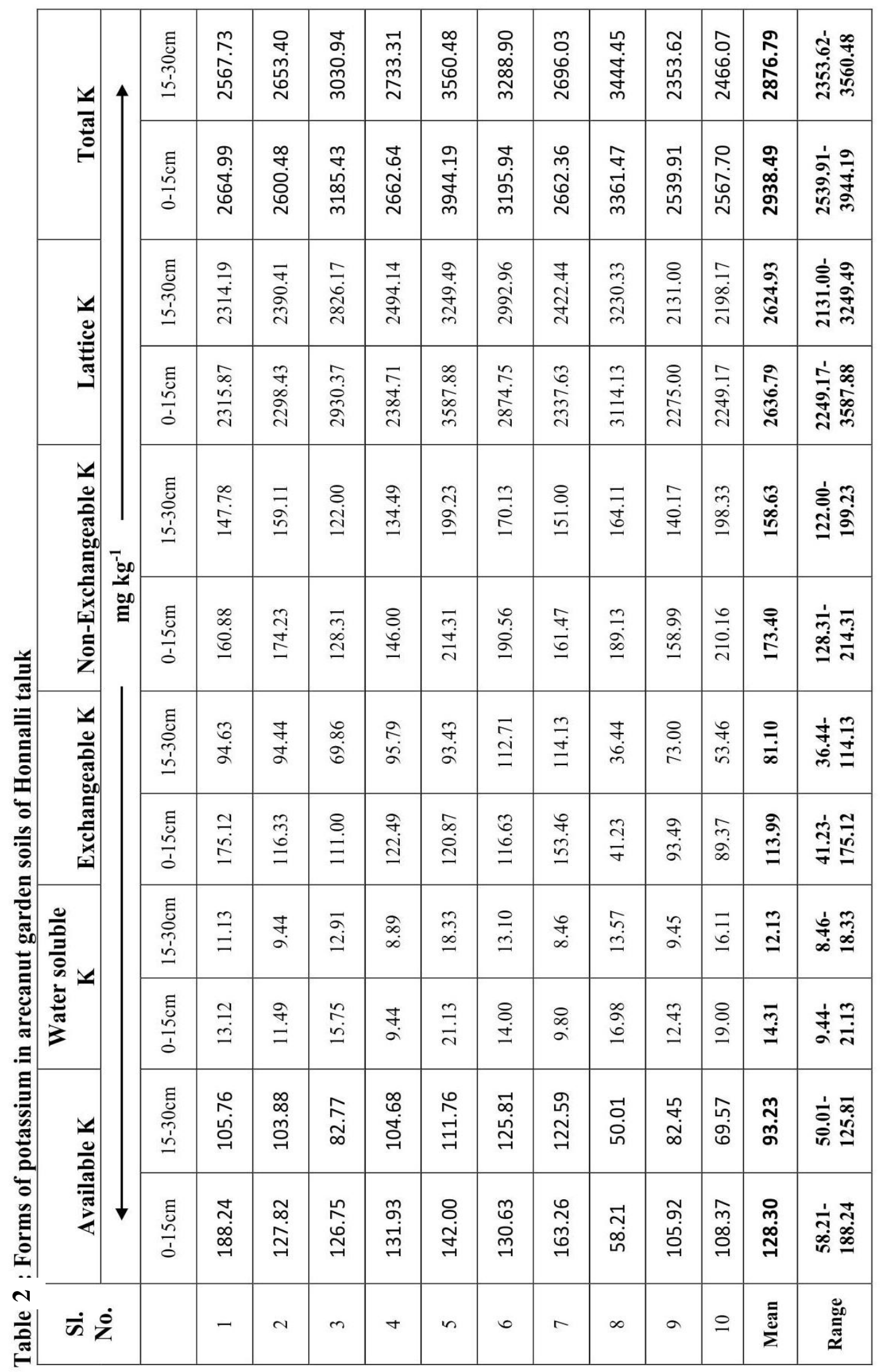




\begin{tabular}{|c|c|c|c|c|c|c|c|c|c|c|c|c|c|c|}
\hline \multirow{2}{*}{$\frac{\Perp}{\vec{\pi}}$} & \multirow{4}{*}{$\uparrow$} & 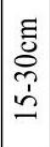 & 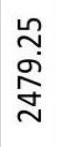 & 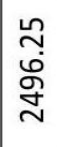 & 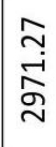 & 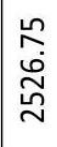 & 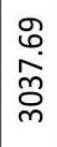 & $\begin{array}{l}0 \\
\tilde{c} \\
\tilde{n} \\
\tilde{m} \\
\tilde{m}\end{array}$ & 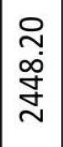 & 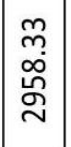 & 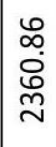 & $\begin{array}{l}\vec{m} \\
\underset{j}{\mathcal{J}}\end{array}$ & 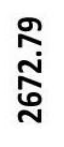 & 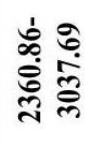 \\
\hline & & $\begin{array}{l}\tilde{\Xi} \\
\stackrel{0}{\circ} \\
\dot{0}\end{array}$ & $\begin{array}{l}8 \\
\infty \\
\infty \\
\stackrel{\infty}{ٍ ٌ}\end{array}$ & 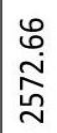 & 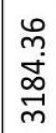 & 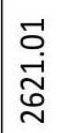 & $\begin{array}{l}\stackrel{0}{\sim} \\
\infty \\
\stackrel{\infty}{2} \\
\underset{m}{2}\end{array}$ & $\begin{array}{l}\hat{N} \\
\dot{j} \\
\vec{m} \\
\vec{m}\end{array}$ & 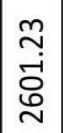 & $\left|\begin{array}{l}\infty \\
-1 \\
\dot{0} \\
0 \\
-1 \\
m\end{array}\right|$ & $\begin{array}{l}\text { ̃ } \\
\tilde{N} \\
\stackrel{N}{N}\end{array}$ & $\begin{array}{l}\underset{\sim}{\sim} \\
\stackrel{\text { ㄱ. }}{N}\end{array}$ & $\begin{array}{l}\text { హ్ } \\
\text { జ్ } \\
\text { స్ }\end{array}$ & 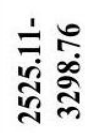 \\
\hline \multirow{2}{*}{ 苞 } & & 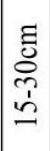 & $\begin{array}{l}\text { 苞 } \\
\text { ते }\end{array}$ & 竞 & 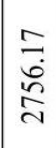 & $\underset{⿱ 亠 凶}{\stackrel{\Xi}{d}}$ & 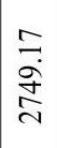 & 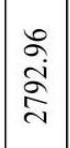 & 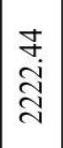 & $\begin{array}{c}= \\
\stackrel{0}{0} \\
\stackrel{n}{\sim}\end{array}$ & $\frac{8}{\frac{8}{m}}$ & $\begin{array}{l}\stackrel{8}{0} \\
\infty \\
\stackrel{\infty}{\sim}\end{array}$ & 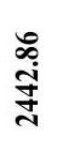 & 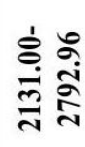 \\
\hline & & $\frac{\Xi}{0}$ & $\begin{array}{l}= \\
\frac{\vec{n}}{2}\end{array}$ & $\begin{array}{l}\stackrel{0}{0} \\
\infty \\
\stackrel{0}{\text { ते }}\end{array}$ & $\begin{array}{l}\bar{n} \\
\text { ָे } \\
\text { ָे }\end{array}$ & $\begin{array}{l}n \\
\tilde{n} \\
\infty \\
\stackrel{\sim}{n}\end{array}$ & $\begin{array}{l}\stackrel{8}{0} \\
\stackrel{+}{\infty} \\
\stackrel{\lambda}{2}\end{array}$ & $\underset{\substack{\infty \\
\underset{+}{+} \\
\underset{\sim}{\infty}}}{ }$ & 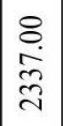 & $\begin{array}{c}8 \\
\dot{T} \\
\dot{T}\end{array} \mid$ & 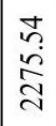 & 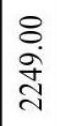 & 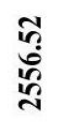 & 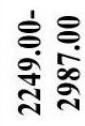 \\
\hline \multirow{2}{*}{ 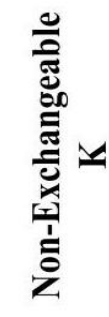 } & \multirow{2}{*}{ T } & 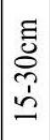 & $\underset{\check{n}}{\stackrel{2}{2}}$ & $=$ & $\begin{array}{l}\text { 二 } \\
\text { I }\end{array}$ & 守 & $\stackrel{\text { f̊ }}{\stackrel{2}{I}}$ & $\begin{array}{l}m \\
\stackrel{0}{0}\end{array}$ & 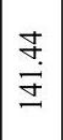 & $\begin{array}{l}\exists \\
\dot{J}\end{array}$ & 吾 & $\begin{array}{l}\infty \\
\infty \\
\infty \\
=\end{array}$ & 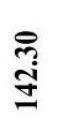 & $\begin{array}{l}\text { लि } \\
\infty \\
0 \\
0\end{array}$ \\
\hline & & $\frac{\Xi}{\tilde{D}}$ & $\begin{array}{l}\infty \\
\stackrel{\infty}{n}\end{array}$ & $\begin{array}{l}\infty \\
\stackrel{+}{+} \\
\stackrel{0}{0}\end{array}$ & $\begin{array}{l}\vec{n} \\
\infty \\
\tilde{n}\end{array}$ & $\begin{array}{l}8 \\
\stackrel{0}{0} \\
\text { I }\end{array}$ & 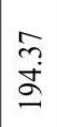 & 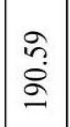 & $\underset{\text { f }}{\stackrel{f}{\dot{m}}}$ & 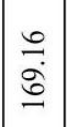 & $\begin{array}{l}2 \\
\alpha \\
\infty \\
\approx\end{array}$ & $\begin{array}{l}\stackrel{m}{ \pm} \\
\stackrel{+}{ \pm}\end{array}$ & 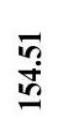 & 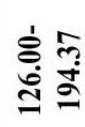 \\
\hline \multirow{2}{*}{ 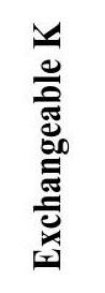 } & & 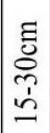 & $\stackrel{7}{\underset{7}{7}}$ & $\stackrel{\infty}{\stackrel{0}{0}}$ & $\frac{1}{1}$ & 8. & \begin{tabular}{l}
$\stackrel{0}{0}$ \\
\multirow{2}{\alpha}{}
\end{tabular} & $\begin{array}{l}8 \\
0 \\
8\end{array}$ & \begin{tabular}{|l|}
8 \\
\\
\end{tabular} & $\begin{array}{c}8 \\
\infty \\
\infty\end{array}$ & $\underset{\infty}{m}$ & $\begin{array}{l}\infty \\
\stackrel{\infty}{\circ}\end{array}$ & ஜึ & 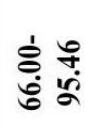 \\
\hline & \multirow{2}{*}{. } & 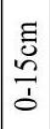 & సี & $\stackrel{m}{\stackrel{m}{\Xi}}$ & స్ & $\vec{m}$ & 灾 & $\begin{array}{l}\infty \\
\infty \\
\dot{\omega} \\
\varrho\end{array}$ & 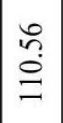 & $\begin{array}{l}\stackrel{2}{+} \\
\dot{\&}\end{array}$ & $\begin{array}{l}a \\
\dot{a} \\
\dot{0}\end{array}$ & 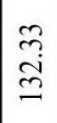 & 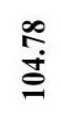 & $\begin{array}{l}\dot{m} \\
\vec{m} \\
\stackrel{2}{2}\end{array}$ \\
\hline \multirow{2}{*}{ 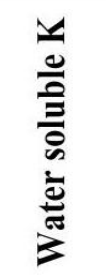 } & & $\begin{array}{l}\tilde{0} \\
\text { Dे } \\
\dot{c} \\
\dot{c}\end{array}$ & స్ర & $\underset{ָ}{\sim}$ & 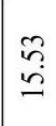 & $\stackrel{7}{0}$ & $\begin{array}{l}\hat{\sigma} \\
\dot{2}\end{array}$ & 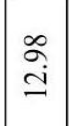 & 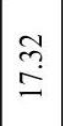 & $=$ & $\stackrel{2}{n}$ & $\underset{\infty}{8}$ & 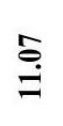 & 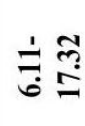 \\
\hline & & $\begin{array}{c}\frac{n}{0} \\
\frac{n}{0}\end{array}$ & $\begin{array}{l}\infty \\
\infty \\
\infty\end{array}$ & $\stackrel{2}{\circ}$ & 年 & $\stackrel{n}{\check{c}}$ & 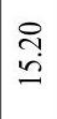 & $\left|\begin{array}{l}\tilde{n} \\
\tilde{n}\end{array}\right|$ & $\begin{array}{l}\text { तิ } \\
\text { הู }\end{array}$ & $\underset{\infty}{\infty}$ & $\begin{array}{l}n \\
\alpha \\
\alpha\end{array}$ & $\underset{\sigma}{6}$ & $\underset{9}{=}$ & 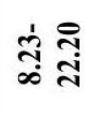 \\
\hline \multirow{2}{*}{ 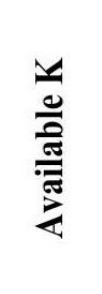 } & \multirow{2}{*}{$\downarrow$} & 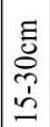 & $\stackrel{\stackrel{n}{m}}{\stackrel{n}{\curvearrowright}}$ & م) & જू & $\underset{\infty}{\stackrel{\sim}{\sim}}$ & $\begin{array}{l}\text { 웅 } \\
\text { gे }\end{array}$ & $\mid \begin{array}{l}\infty \\
\stackrel{\infty}{+} \\
\stackrel{\infty}{1}\end{array}$ & $\begin{array}{l}\tilde{m} \\
\tilde{\infty} \\
\dot{\infty}\end{array}$ & $\mid \begin{array}{l}\vec{I} \\
\overrightarrow{\dot{D}} \\
\dot{\infty}\end{array}$ & $\begin{array}{l}0 \\
0 \\
\infty \\
\infty\end{array}$ & $\begin{array}{l}\infty \\
\stackrel{2}{\alpha} \\
\end{array}$ & 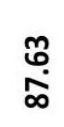 & $\begin{array}{l}\stackrel{2}{\circ} \\
\stackrel{\circ}{\circ}\end{array}$ \\
\hline & & $\begin{array}{l}\frac{z}{0} \\
\frac{n}{o}\end{array}$ & 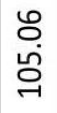 & 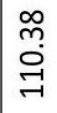 & $\begin{array}{l}\text { N } \\
\text { In } \\
\text { I }\end{array}$ & \begin{tabular}{l}
0 \\
\multirow{+}{0}{} \\
$\stackrel{0}{-1}$
\end{tabular} & 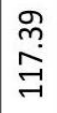 & $\begin{array}{l}\text { 우 } \\
\text { ğ } \\
\end{array}$ & $\begin{array}{l}\stackrel{0}{\stackrel{v}{i}} \\
\underset{\sim}{\sim}\end{array}$ & $\begin{array}{c}\tilde{O} \\
\dot{m} \\
\stackrel{-}{1}\end{array} \mid$ & 咅 & 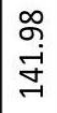 & $\begin{array}{l}\stackrel{\circ}{\infty} \\
\text { ને }\end{array}$ & 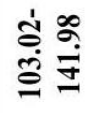 \\
\hline \multicolumn{2}{|l|}{$\begin{array}{l}\dot{z} \\
\dot{n}\end{array}$} & & - & $N$ & $m$ & 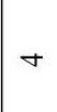 & in & 0 & $r$ & $\infty$ & $a$ & 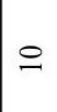 & 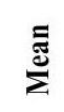 & 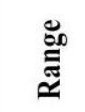 \\
\hline
\end{tabular}




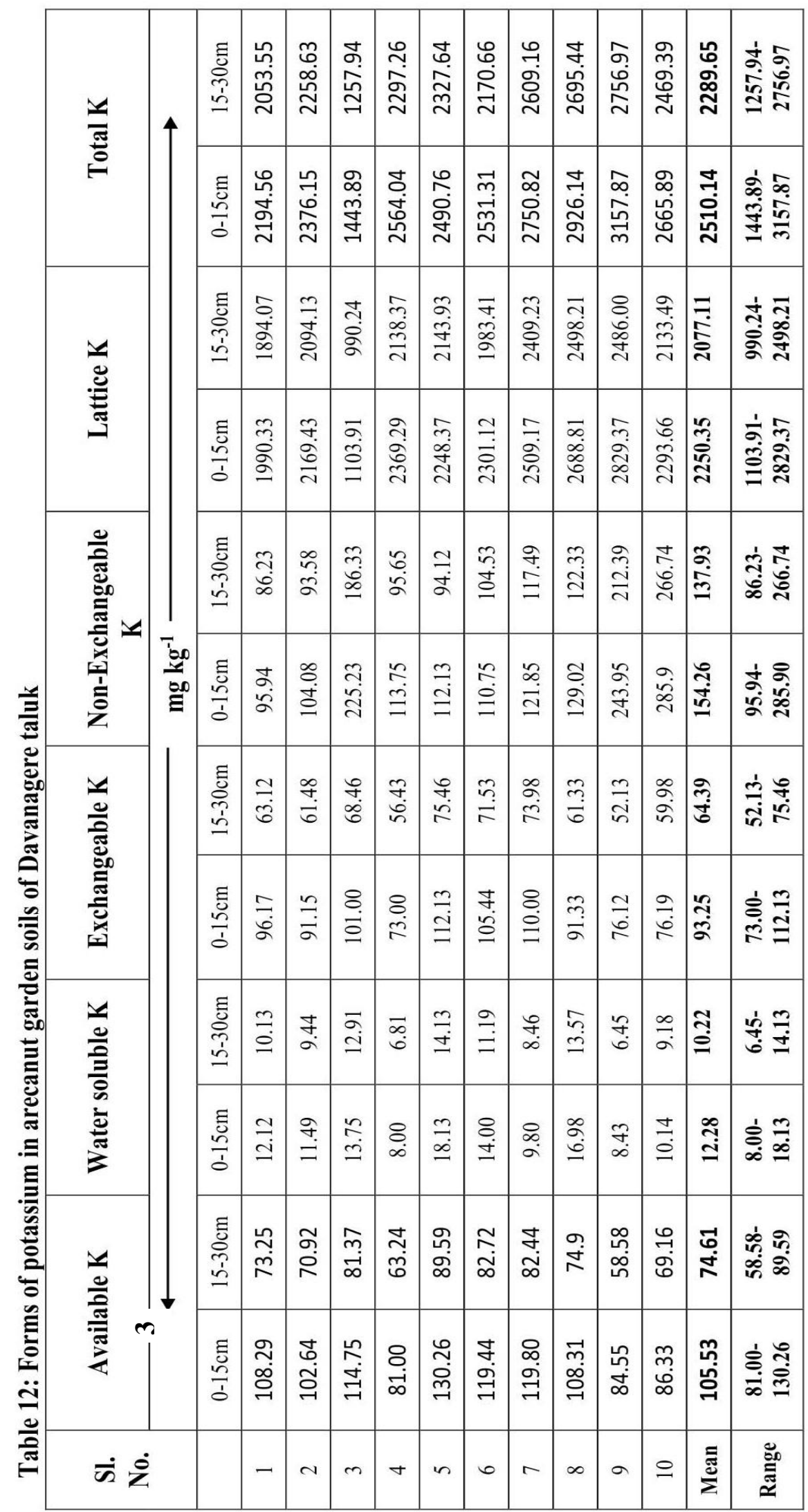


In sub-surface soils, the non-exchangeable potassium content varied from 86.23 to $266.74 \mathrm{mg} \mathrm{kg}^{-1}$, this form of potassium varied from 1103.91 to $2829.37 \mathrm{mg} \mathrm{kg}^{-1}$ in the surface and from 990.24 to $2492.21 \mathrm{mg} \mathrm{kg}^{-1}$ in the sub-surface soils. The total potassium content varied from 1443.89 to $3157.87 \mathrm{mg}$ $\mathrm{kg}^{-1}$ and 1257.94 to $2756.97 \mathrm{mg} \mathrm{kg}^{-1}$ in surface and sub-surface soils, respectively.

Range of available potassium is medium to high in all the taluks. Coarse textured soils recorded comparatively low available potassium status than heavy textured soils. The higher $\mathrm{K}$ values in heavy textured soils could be due to predominance of $\mathrm{K}$ rich micaceous and feldspars minerals in these soils. These results are in agreement with the findings of Ravikumar (2006) who recorded higher available potassium status in Vertisols compared to Alfisols. The surface soils contained higher potassium content than subsurface soils. This could be attributed to more intense weathering, release of potassium from organic residues, application of potassium fertilizers and upward translocation of potassium from lower depth along with capillary rise of ground water. Similar findings were made by Varaprasad Rao et al., (2008) (Table 1 to 4 ).

The water soluble potassium content was lower in the sub-surface layer than in surface layer of soils. This might be due to intense pedochemical weathering, higher organic carbon content and release of labile $\mathrm{K}$ from organic residues as suggested by Raskar and Pharande (1997). The reasons for higher concentration of water soluble $\mathrm{K}$ in surface soils may be due to the addition of fertilizers, presence of potassium bearing minerals, intensity of weathering and upward movement of soluble potassium from the lower layers due to capillary rise of ground water as reported by Ranganathan and Satyanarayana (1980), Sudharmai Devi et al.,
(1990) in soils of different agro climatic zones of Karnataka and Kerala, respectively (Table 1 to 4$)$.

The soils of Channagiri taluk contained higher amount of exchangeable potassium than other taluks. The soils differ in exchangeable potassium and such variations in different soils were reported by Sonar and Patil (1996). The exchangeable potassium status of Davanagere soils was comparatively low indicating the dominance of kaolinitic type of clay mineral. Similar findings were recorded by Zubillaga and Conti (2009) in red and laterite soils of Karnataka. Lower content of non- exchangeable potassium in soils of Davanagere taluk attributed to depletion of available potassium from soils by crop removal. Arecanut being a deep rooted and perennial crop might have utilized nonexchangeable potassium in sub-surface soils as compared to surface soils. Similar results were obtained by Vijay Kumar et al., (1986). There is linear relationship between the amount of clay and non- exchangeable potassium and hence the Channagiri soils have high non- exchangeable potassium due to high clay content. This clearly indicates the influence of climate besides the variations in parent material for high content of nonexchangeable potassium in Channagiri soils. Sonar and Patil (1996) were also of the same opinion for the variations in nonexchangeable potassium content for different soils of Maharashtra (Table 1 to 4 ).

In general, lattice potassium contributed highest to the total potassium in all the soils. The highest content of lattice potassium in Channagiri soils could be due to its parent material. The variation in lattice potassium content is due to the abundance or insufficiency of $\mathrm{K}$ - bearing primary and secondary minerals as suggested by Hirekurabar et al., (2000). According to Gangopadhyay et al., (2005), the lattice 
potassium constituted on an average of 91 per cent of total potassium in some soils of Karnataka. The contribution of different forms of potassium to total potassium in soils of different taluks under study can be arranged in the order of water-soluble potassium< exchangeable potassium $<$ nonexchangeable potassium < lattice potassium. The wide variation in total potassium may be attributed to the preponderance or insufficiency of potash bearing minerals and particle size. Similar results were reported by Gangopadhyay et al., (2005). The high content of total potassium in Channagiri soils could be due to the presence of potash bearing minerals viz., mica and feldspar. Davanagere taluk soils had recorded low content of total potassium which might be due to the dominance of kaolinite clay fraction. Similar results were obtained by Jagadeesh (2003) for coastal soils of Karnataka (Table 1 to 4).

The area under arecanut cultivation in Davanagere district has increased, nut cracking in arecanut is a nutritional disorder caused due to potassium deficiency. Analysis of nutrient status in soils may throw light on the variation in nutrient compositions. In the present study, nutrient rating was found in the range of medium to high in most of all soils, which shows potassium status in the arecanut gardens was good and helps in better management of nutrients in different arecanut growing areas of Davanagere district. The morphological studies of surface and subsurface soils showed that the soils of all the taluks are well drained and hence the incidence of diseases is least.

\section{References}

Badrinath, M. S., Krishnan, A. M., Patil, B. N., Kenchaiah, K. and Balakrishna Rao, K. 1996. Fertility status of some typical soils of coastal Karnataka. J. Indian Soc. Soil Sci., 34: 436-438.
Black, C. A. 1965. Methods of Soil Analysis Part - II, Agronomy Monograph 9, American Society of Agronomy, Madison, Wisconsin, USA.

Chandel, A. S., Singh, M. and Singh, T.A. 1976. Bulletin. Indian Society of Soil Science, 13: 20.

Gangopadhyay, S. K., Bhattacharya, T. and Sarkar, D. 2001. Rubber growing soils of Tripura their characteristics and classification. J. Indian Soc. Soil Sci., 49(1): 164-170.

Hirekurabar, B. M., Satyanarayana, T., Sarangamath, P. A. and Manjunathaiah, H. M. 2000. Forms of potassium and their distribution in soils under cottonbased cropping system in Karnataka. $J$. Indian Soc. Soil Sci., 48: 604-608.

Jackson, M. L. 1973. Soil Chemical Analysis. Prentice Hall of India (Pvt.) Ltd., New Delhi.

Jagadeesh, B. R. 2003. Dynamics of potassium in soils of selected agro climatic zones of Karnataka. Ph.D. (Thesis). Univ. Agril. Sci., Bangalore.

Page, A.L, Miller, R.H. and Kenay, D.R. 1982. Methods of Soil Analysis, part 2, Soil Science Society of America, Inc, Publishers, Madison, Wisconsin, USA.

Piper, C. S. 2002. Soil and Plant Analysis, Hans Publishers, Bombay, p. 368.

Ranganathan, A. and Satyanarayana, T. 1980. Studies on potassium status of soils of Karnataka. J. Indian Soc. Soil Sci., 28(2): 148-153.

Raskar, B.N. and Pharande, A.L. 1997. Different forms of potassium and their distribution in some important soil series of Vertisol and Alfisol of Western Maharashtra. J. Potassium.Res., 13(1): 21-30.

Sonar, K. R. and Patil, Y. M. 1996. Forms of potassium as influenced by geology and physiography of soils of Maharashtra. $J$. Potassium. Res., 12(3): 230-236. 
Sparks, 1996. Methods of soil analysis, Part 3: Chemical Methods, Soil Science Society of America, USA.

Sudharmai Devi, C.R., Korah, P.A., Usha, P.B. and Saraswathi, P. 1990. Forms of potassium in two soil series of south Kerala. J. Potass Res., 6: 9-15.

Tandon, H.L.S. and Narayan, P. 1990. Fertilizers in Indian agriculture - past, present and future (1950-2000). Fertilizer Development and Consultation Organization, New Delhi. pp. 160.

Varaprasad Rao, A. P., Naidu, M. V. S., Ramavatharam, N. and Rama Rao, G.
2008. Characterization, classification and evaluation of soils on different landforms in Ramachandrapuram mandal of Chittoor district in Andhra Pradesh for sustainable land use planning. J. Indian Soc. Soil Sci., 56(1): 23-33.

Zubillaga, M. M. and Conti, M. 2009. Importance of textural fraction and its mineralogical characteristic in the potassium contents of different Argentia of soils commun. Soil Sci. Pl. Anal., 25(5\&6): 479-487.

\section{How to cite this article:}

Amruthesh, P., Parashuram Chandravamshi, K.T. Gurumurthy, L.B. Ashok, D.A. Sumana and Narayan S. Mavarkar. 2018. Status and Distribution of Potassium in Soils under Arecanut Gardens of Bhadra Command Area of Davanagere District, India. Int.J.Curr.Microbiol.App.Sci. 7(12): 288-297. doi: https://doi.org/10.20546/ijcmas.2018.712.035 